\title{
Diagnóstico de linfangioma cavernoso de face em paciente adulto: relato de caso clínico.
}

\author{
Diagnosis of Cavernous Lymphangioma of the face \\ in adult patient: report of a case.
}

\author{
Prof ${ }^{\text {f. }}$ Dra. Edela Puricelli* \\ CD Juliana Romanini** \\ CD Júlio César Munerato** \\ CD Márcia Gaiger de Oliveira**
}

\begin{abstract}
RESUMO
Os autores apresentam um caso clínico de paciente do sexo masculino, 18 anos, portador de lesão recidivante de crescimento expansivo intra e extrabucal, na face esquerda, comprometendo a mucosa jugal. O tumor revelou-se indolor, associado à progressiva limitação dos movimentos mandibulares. O diagnóstico clínico e histopatológico de linfangioma cavernoso diferiu do diagnóstico por ressonância magnética.
\end{abstract}

\section{UNTTERMOS}

Linfangioma cavernoso, adulto, recidivante, ressonância magnética, biópsia.

\section{INTRODUÇÃO}

A origem dos linfangiomas ainda é incerta. Muitos autores consideram-os lesões neoplásicas benignas de vasos linfáticos e tecido glandular, determinando uma forma de angiodisplasia inativa ou de circulação lenta ${ }^{8}$. 10. 12. 17. 19.21, 23 . Ao mesmo tempo, aceitam a possibilidade de que sejam processos de malformaçāo ou hamartomas. A origem destes, então, estaria na seqüestração de tecido linfático isolado e privado de suas conexões com o sistema venoso ${ }^{2,5,20}$.

Em 1877, WEGNER apresentou a classificaçāo histológica até hoje aceita, baseada na dimensão dos canais linfáticos da lesão $\%, 17$, ${ }^{21}$. O linfangioma cístico ou higroma é o tipo histológico mais comum. Apresenta as maiores dilataçôes dos canais linfáticos, exibindo espaços císticos macroscopicamente bem circunscritos. Ao exame microscópico, seus espaços císticos são limitados por células endoteliais planas e por delgadas camadas de músculo liso contidos numa matriz de tecido conjuntivo. $O$ linfangioma cavernoso apresenta vasos linfáticos grandes e dilatados. Geralmente não tem os canais bem circunscritos e possui menor quantidade de músculo liso. Muitas vezes o linfangioma cavernoso tem uma adventícia fibrosa ${ }^{10}$. O linfangioma capilar ou simples é muito raro. Tem canais linfáticos pequenos e comprimidos e ausência de músculo liso 9, 17.21 .

Baseando-se na teoria centrípeta ou aferente, o higroma cistico origina-se da falha dos espaços linfáticos primários que confluem para o sistema central ${ }^{21}$. Já no cavernoso, a seqüestraçāo tecidual é mais periférica que no higroma; os espaços linfáticos primários não confluem para os coletores centrais principais. O linfangioma capilar resulta de uma perda de liquidos ou seqüestração tecidual localizada nas poucas fendas mesenquimais. Finalmente, o hemangio-linfangioma é composto por vasos linfáticos e sangüíneos ${ }^{9,21}$.

Os linfangiomas podem ser detectados

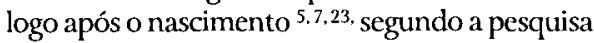
de Sabin (1902) e van der Putte (1975) que revelaram que o sistema linfático se desenvolve durante a sexta semana da embriogêse, o que pode explicar o aparecimento tão precoce das lesões ${ }^{9}$. Sabe-se que $50 \%$ dos casos aparecem ao nascer e cerca de $90 \%$ desenvolvem-se, em média, até os dois anos de idade. Isso se deve, provavelmente, por corresponder ao período de maior desenvolvimento do sistema linfático". 11, 12,17. Entretanto, há relato do aparecimento de lesōes durante as primeiras décadas de vida ${ }^{5}$, 20.23. Homens e mulheres são afetados com igual freqüência $7.9 .13,18,26,30$.

Aproximadamente $\quad 75 \%$ dos linfangiomas podem localizar-se na face e região cervical 4. 13. 17.31. Os linfangiomas simples e cavernosos afetam zonas onde o tecido conjuntivo é compacto. Na cavidade bucal são encontrados na língua, no palato, gengiva, lábios e mucosa jugal 1.3. 5. 6.8. 14, 18. 19.23. Na língua, atingem a região do ápice dorsal e ventral ${ }^{5.13 .18}$; quando localizados profundamente, produzem macroglossia linfomatosa ${ }^{3,6,8,18,27}$; e envolvendo o lábio, podem levar à macroqueilia 5.8 . Em recém-nascidos da raça negra, os linfangiomas ocorrem no rebordo alveolar, assumindo aspecto próprio, com cápsula azulada e apresentando remissão espontânea ${ }^{17}$. O linfangioma cístico tem preferência por áreas onde o tecido conjuntivo é mais frouxo, como na regiāo cervical. Em alguns casos, os linfangiomas ocorrem de forma difusa, denominada linfangiomatose, comprometendo face, pescoco, boca ou extremidades $\%, 21$.

Os linfangiomas podem ser profundos ou superficiais. Quando localizados profundamente, apresentam-se como massas submucosas ou nódulos difusos sem alteraçōes significativas de textura ou cor na regiăo. As lesōes superficiais produzem tumefações nodulares semelhantes a vesículas, formando múltiplas excrescências moles, compressíveis e assintomáticas. Geralmente são translúcidas, de coloração similar à mucosa ou ligeiramente mais avermelhadas 5, 8, 17, 20, 21, 23, 30. Essas vesículas são preenchidas por linfa. Eventualmente, quando traumatizadas, podem conter sangue, tornando-se escurecidas. Essa alteração local leva o paciente a buscar esclarecimento de um profissional ${ }^{5}$. Durante o exame clínico, à palpação, pode-se observar o deslocamento da linfa de um ponto para outro, provocando crepitação ${ }^{5.7}$.

O exame por imagem é indispensável para localização e diagnóstico diferencial da lesão. A tomografia computadorizada é limitada. A ressonância magnética $(\mathrm{RM})$ apresenta melhor resoluçāo para tecidos moles. $\mathrm{Na} \mathrm{RM}$, o conteúdo líquido que deverá ser predominante nas cavidades císticas é revelado a partir da intensidade de sinais, a qual é menos intensa nas imagens em TIWI e hiperintensa nas imagens T2WI. A natureza múltipla e intercomunicante dos cistos e qualquer nível fluido-fluido são melhores ponderados em T2WI. Segundo FRASER, a TC ou a RM em T2, podem

\footnotetext{
* Doutora pela Universidade de Düsseldorf - Alemanha; Chefe da Unidade de CTBMF do Hospital de Clínicas de Porto Alegre - HCPA; Profa da Disciplina de Patologia Cirúrgica - Mestrado em Odontológica Concentração Patologia Bucal - Faculdade de Odontologia - UFRGS.

** Mestrandos em Odontológica Área de Concentração Patologia Bucal - Faculdade de Odontologia - UFRGS.
} 
diferenciar muito bem uma massa sólida de uma cística, sendo que ambas facilitam a confirmação e localização da lesão. As radiografias, muitas vezes, são utilizadas como primeira opção de exame nas patologias faciais. Especialmente nos tumores de tecidos moles, servem para revelar envolvimento secundário, relacionado principalmente com as estruturas dentárias, sinusais e articulação temporomandibular (ATM). A ecografia ou sonografia pode ser indicada para lesões tumorais superficiais.

$\mathrm{O}$ aspecto clínico de linfangioma pode levar a um diagnóstico inicial de hemangioma lesão proliferativa de vasos sangüíneos ${ }^{6.23}$. Podese então, realizar o exame por angiografia, que permitirá, nos casos de hemangioma, determinar o suprimento sangüineo e drenagem destes tumores ${ }^{31}$, além da localização e extensão dos mesmos. Ainda no diagnóstico diferencial, devese levantar as possibilidades de lipoma, teratoma e mucocele. Especialmente na região cervical, lembramos cistos dermóide, branquial ou do tiroglosso ${ }^{21}$. Na região orbital devem ser afastados os neurofibromas e na regiāo occipital o meningoencefalocele ${ }^{21}$.

Os linfangiomas têm crescimento lento, com tendência à estabilização. Não se observam a involução das lesões 5. 8.20.23 ou a transformação maligna ${ }^{3.4}$, apesar da decorrência de tempo. Após os diagnósticos clínicos, é indispensável a biópsia para o exame histopatológico ${ }^{8}$.

Microscopicamente, nos linfangiomas, observam-se massas de vasos linfáticos dilatados e preenchidos por linfa. As paredes vasculares são delgadas e formadas por células endoteliais. Nas lesōes superficiais, estes espaços situam-se imediatamente abaixo do epitélio ${ }^{1.3,5,14,16}$. Os vasos podem apresentar grandes agregados linfóides em suas paredes e, eventualmente, podem ser observadas hemácias em seu interior, em função de hemorragia secundária ${ }^{17}$.

Os linfangiomas apresentam inúmeras possibilidades de tratamento, tais como excisão cirúrgica, crioterapia e esclerose ${ }^{5.10,19,20,21.24} \mathrm{e}$ ainda a eletrocoagulaçăo e o laser ${ }^{4.19}$. A radioterapia já há muito tempo não é mais usada, devido a sua inabilidade na completa destruição tumoral e seu potencial de malignização ${ }^{12,19,21}$. As inúmeras propostas terapêuticas servem para definir estas lesóes como as mais complexas no tratamento buco-maxilofacial ${ }^{13,21}$

\section{CASO CLÍNICO}

O paciente R.F, 18 anos, sexo masculino, branco, procurou atendimento na Disciplina de Cirurgia e Traumatologia Buco-maxilo-faciais da Faculdade de Odontologia da Universidade Federal do Rio Grande do Sul-UFRGS, para diagnóstico e tratamento de volume indolor na face, lado esquerdo, associado à progressiva limitação dos movimentos mandibulares. Durante a anamnese, o paciente referiu que aos 13 anos de idade foi submetido à intervenção cirúrgica na região. $O$ diagnóstico pré-operatónio foi de tumor de vasos sangüíneos (sic). O próprio paciente e familiares não detinham maiores conhecimentos sobre o tratamento realizado.

No exame clínico extrabucal, além de importante volume abrangendo a hemiface esquerda, comprovou-se a limitação mecânica dos movimentos mandibulares. $\mathrm{Na}$ pele visualizava-se a cicatriz da incisão tipo WeberFergunson (Fig. I). Não foram detectadas adenopatias cervicais ou sinais clínicos sistêmicos de infecção.

Já no exame intrabucal, clinicamente observou-se aumento de volume com alteração da anatomia jugal esquerda e presença de inúmeras pequenas vesículas variando desde a coloração normal da mucosa, passando pela avermelhada, até vermelho escuro (Fig.2). A apalpação bidigital, a lesão apresentava-se nodular, de consistência densa, sem referência de sintomatologia dolorosa. Clinicamente, as demais estruturas intrabucais não apresentavam comprometimento com a lesão $O$ paciente não relatava queixas quanto a dificuldade de deglutição, fonação ou disfagia. O diagnóstico presuntivo foi de linfangioma.

O paciente portava exame de angiografia arterial de carótida externa e interna, sendo afastado o diagnóstico de lesão hemangiomatosa. $\mathrm{O}$ laudo da ressonância magnética (RM) de encéfalo e face relatou lesão expansiva heterogênea, de contornos irregulares, comprometendo o espaço retromaxilar. A lesão envolvia, ainda, a porção superior do ramo e côndilo mandibulares esquerdos. Cranialmente, o tumor se estendia pela fossa temporal e, em direçāo medial, deformava a parede lateral do seio maxilar (Fig.3). Nāo houve evidências de invasão do seio maxilar, nem das demais estruturas ósseas limitantes do processo tumoral. Na injeção do contraste TI, a lesão apresentouse de intensidade levemente superior ao tecido muscular; em T2 foi hiperintensa, e após a injeção do contraste para-magnético, impregnou-se heterogeneamente, especialmente na periferia. A descriçāo da imagem é sugestiva de diagnóstico de linfangioma provavelmente do tipo cístico.

O paciente foi então submetido à biópsia incisional, sob efeitos de anestesia local. Aárea explorada foi a regiāo jugal, envolvendo a mucosa em superfície e profundidade compativeis para o preparo histopatológico.

As imagens microscópicas revelaram espaços vasculares dilatados, que mostraram tendência a ocupar porçóes subepiteliais, contendo material hialino com células inflamatórias (Fig.4). Em alguns vasos observaram-se hemácias. Como resultado, obteve-se o diagnóstico de Linfangioma Cavernoso.

\section{DISCUSSÃO}

Os linfangiomas são pouco freqüentes na idade adulta. Segundo QIN (1998), a probabilidade do aparecimento deste tumor é de $10,5 \%$ em paciente entre 10 e 20 anos de idade. Já em pacientes em idade superior a 20 anos, a freqüência baixa para $9 \%$. Em sua publicação, HERRERA (1998) apresenta relatos clínicos de dois pacientes com 18 e 59 anos. Tal condição amplia significativamente a faixa etária para diagnóstico do tumor. Por isso, quando indivíduos adultos apresentam tumoraçōes na face, a suspeita clínica de linfangioma deve ser lembrada. Para FRASER (1996), apenas $12,5 \%$ dos linfangiomas em pacientes adultos foram presuntivamente diagnosticados no exame clínico.

Uma explicação para o aparecimento tardio destes tumores é defendida por SAIJO (1975), baseada na desproporcionalidade entre o cresciemnto físico do paceinte e do tumor. Entretanto, o registro destas lesões predominantemente na infância mantém a teoria das proporcionalidades como o conceito próprio.

Quanto à localização anatômica, os linfangiomas podem apresentar cinco diferentes estágios. Esta classificação proposta por SERRES (1995) usa como referência o osso hióide, com distribuição superior e/ou inferior em uni e bilateralidade.

SERRES

Quadro I: Classificação segundo

\begin{tabular}{|c|c|c|}
\hline Estágio & $\begin{array}{c}\text { Localização } \\
\text { da lesão }\end{array}$ & $\begin{array}{c}\text { Latera- } \\
\text { lidade }\end{array}$ \\
\hline I & $\begin{array}{c}\text { Infra- } \\
\text { hioidea }\end{array}$ & Unilateral \\
\hline II & $\begin{array}{c}\text { Supra- } \\
\text { hioidea }\end{array}$ & Unilateral \\
\hline III & $\begin{array}{c}\text { Supra e infra- } \\
\text { hioidea }\end{array}$ & Unilateral \\
\hline IV & $\begin{array}{c}\text { Supra- } \\
\text { hioidea }\end{array}$ & Bilateral \\
\hline V & $\begin{array}{c}\text { Supra e infra- } \\
\text { hioidea }\end{array}$ & Bilateral \\
\hline
\end{tabular}

Segundo esta classificação, a lesão descrita é supra-hioidea e unilateral, correspondendo ao estágio II. Geralmente, pacientes com lesão unilateral $\mathrm{e}$ infra-hioidea estágio I tem melhor prognóstico que os com tumores no estágio II. $\mathrm{Na}$ progressão dos prognósticos severos, encontramos os estágios IV $\mathrm{e}$, respectivamente.

$\mathrm{Na}$ face, a cadeia linfática parotídean corresponde a três grupos de distribuiçāo, relacionados com a aponeurose superficial e a glândula parótida. São eles o pré-auricular ou supra-aponeurótico, o infra-auricular ou subaponeurótico e o parotídeo profundo ou intraglandular. $\mathrm{O}$ sistema infra-auricular distribui-se pelos vasos linfáticos provenientes da região geniana, mucosa jugal, nariz, lábio superior e a glândula parótida. Os linfonopos deste sistema podem ainda estar conectados com as cadeias retroauricular e intraglandular parotídea.

Esta complexa anatomia circulatória específica explica a extensão do processo tumoral linfático, o qual pode envolver estruturas intrabucais, espaços medulares e tecidos 
glandulares. Especialmente no caso clínico relatado, encontramos tecido linfático tumoral comprometendo a hemiface esquerda nos seus aspectos extra e intrabucais. Normalmente, os linfangiomas têm crescimento lento, indolor e flutuante. Quando atingem grandes dimensões, criam assimetrias ou deformaçôes extensas. Para SMITH (1996), seu crescimento enquanto ativo infiltra tecidos circunvizinhos, invadindo ou comprimindo estruturas funcionais e vitais. No caso relatado, o paciente apresenta queixa de progressiva limitação dos movimentos mandibulares, provocados pela continuidade do crescimento tumoral.

Segundo QIN (1998), especialmente na região parótida-masseteriana, $o$ aparecimento de linfangiomas obedece uma freqüência de $9 \%$ dos casos. Ainda para SERRES (1995), na área correspondente ao estágio II, dos linfangiomas diagnosticados 35\% foram higromas e $65 \%$ linfangiomas cavernosos.

O hemenagioma é a primeira opção de diagnóstico diferencial. Segundo SOM e BRANDNEIN (1996) e YONETSU (1993), este tumor, quando localizado na face, tem alta incidência na glândula parótida. Especialmente o hemangioma cavernoso, além de freqüente na infância, pode acontecer em pacientes com mais de 16 anos de idade. Devido a esta estreita relação de características clínicas, para o diagnóstico inicial fazem-se necessárias respostas através de exames por imagem.

A angiografia, segundo YONETSU (1993), permite visualizar a nutrição e a drenagem vascular do hemangioma, sem entretanto demonstrar a relação entre a lesão e os tecidos circunvizinhos. Na prática, esta forma de diagnóstico serve para afastar imediatamente a lesão vascular sangüínea da linfática.

A ultrassonografia permite acesso rápido a informações por imagem, podendo o exame ser feito no próprio consultório. Tem ainda a vantagem de produzir uma radiação não ionizante. Entretanto, para KAZIM (1992), FUNG (1998) e YONETSU (1993), sua limitação básica está na profundidade da lesão e interferências de outros tecidos que impedem a obtenção da imagem, situação comum na face.

KAZIM (I 992) e FUNG (1998) consideram que na TC o tumor geralmente aparece homogêneo com a densidade dos tecidos moles. É possível visualizar os limites das áreas infiltradas, mas a definição de delicadas estruturas no tecido mole é limitada. A TC com contraste venoso define melhor a localização anatômica dos tecidos vizinhos e a relação do linfangioma com os vasos sangüíneos.

SIEGER foi quem primeiro publicou a utilização da $R M$ para avaliação de linfangiomas císticos em crianças. Esse exame oferece imagens de alta sensibilidade, tanto para as densidades teciduais como fluidas. Para KAZIM (1992), FUNG (1998) e YUH (1990), a RM produz a informação pré-operatória mais bem detalhada do linfangioma e estruturas vizinhas, que acabam por coincidir com os achados intra-operatórios. A imagem típica na
RM inclui sinal de alta intensidade nas imagens em T2WI e baixa em TIW1. Com sua menor radiação ionizante, tem grande versatilidade nas áreas cervicais e faciais, onde os linfangiomas, especialmente o cístico, comprimem e invadem as estruturas vitais.

Para FUNG (1998), este exame pode também auxiliar na avaliação de estados infecciosos ou hemorrágicos intratumorais. Por sua vez, SIEGER refere ser esta a forma de imagem a mais apropriada no pré-operatório. Lembramos que uma imagem detalhada, dando a extensão, tamanho e localização da lesão, é indispensável para a indicação dos diferentes tipos de biópsia, melhor opção de tratamento e, ainda, para afastar futuros questionamentos quanto ao diagnóstico. No caso apresentado, o exame por RM, se não comprovado por biópsia, conduziria a um diagnóstico de linfangioma cístico. Visto tratar-se de uma lesão já anteriormente submetida a tratamento cirúrgico, certamente produziriam-se alteraçôes tanto na estrutura física quanto nas reparaçōes cicatriciais, o que confunde o diagnóstico por imagem. O resultado da biópsia quando o tecido é obtido na área central da lesão, aqui discutida, perde sua credibilidade pelas mesmas razões já descritas. A opção da coleta tecidual, neste caso, pareceu-nos mais indicada em área patológica ainda íntegra e de fácil acesso.

Mas segundo SMITH (1996), SOM (1996) e YUH (1990), pode também haver sobreposição entre os tipos de linfangiomas, inclusive dos três tipos de tumores que poderāo se sobrepor na mesma massa. Nossa indicação de área a ser biopsiada concorda com os autores, uma vez que seu diagnóstico pode ser estabelecido pela natureza do tecido que o circunda.

A biópsia por aspiração tem indicaçōes no diagnóstico préoperatório. Para QIN (1998), os linfangiomas cavernosos, quando puncionados, não vertem líquido. A biópsia por punção guiada pela ultrassonografia é uma boa indicação nas áreas superficiais da face. Tal conceito pode ser mantido para a biópsia incisional que obtém o tecido tumoral nos seus limites mais externos.

Para MARAN e PEREZ ORBAN apud HERRERA (1998), o prognóstico não depende da natureza do histológico, mas sim das características locais e invasivas do próprio tumor. A remoção cirúrgica completa, segundo FRASER (1996), FONKARLSRUD (1974) e QIN (1998), é a conduta ideal nos pacientes adultos. Entretanto, especialmente no linfangioma cavernoso que se infiltra nos tecidos circunvizinhos, a remoção cirúrgica é muito difícil e a recidiva é maior que $50 \%$.

Segundo BRENNAN (1997) e BÓRAKS (1996), eventualmente os tumores não podem ser removidos completamente por envolverer estruturas vitais. Se não houve: comprometimento orgânico, o paciente convive com o tumor. $\mathrm{Na}$ nossa experiência, ha possibilidades de reintervenção periódicas à distância, segundo evolução do caso.

\section{CONCLUSÃO}

Os linfangiomas cavernosos da face são mais freqüentes na posição supra-hioidea unilateral.

As lesões manipuladas cirúrgica ou terapeuticamente têm suas estruturas física e histológicas alteradas, comprometendo os exames por imagem e histopatológicos.

Os linfangiomas são menos freqüentes em adultos e devem ser diferenciados. principalmente, de hemngiomas cavernosos.

A possibilidade da presença de linfangioma cístico, cavernoso e capilar em uma mesma massa tumoral pode levar a diagnóstico impreciso.

No diagnóstico por imagem, a RM é o exame mais indicado; entretanto, deve ser complementado por exame histopatológico da lesão.

O material obtido por biópsia incisional poderá ser removido das áreas mais externas e superficiais, principalmente em casos de recidiva.

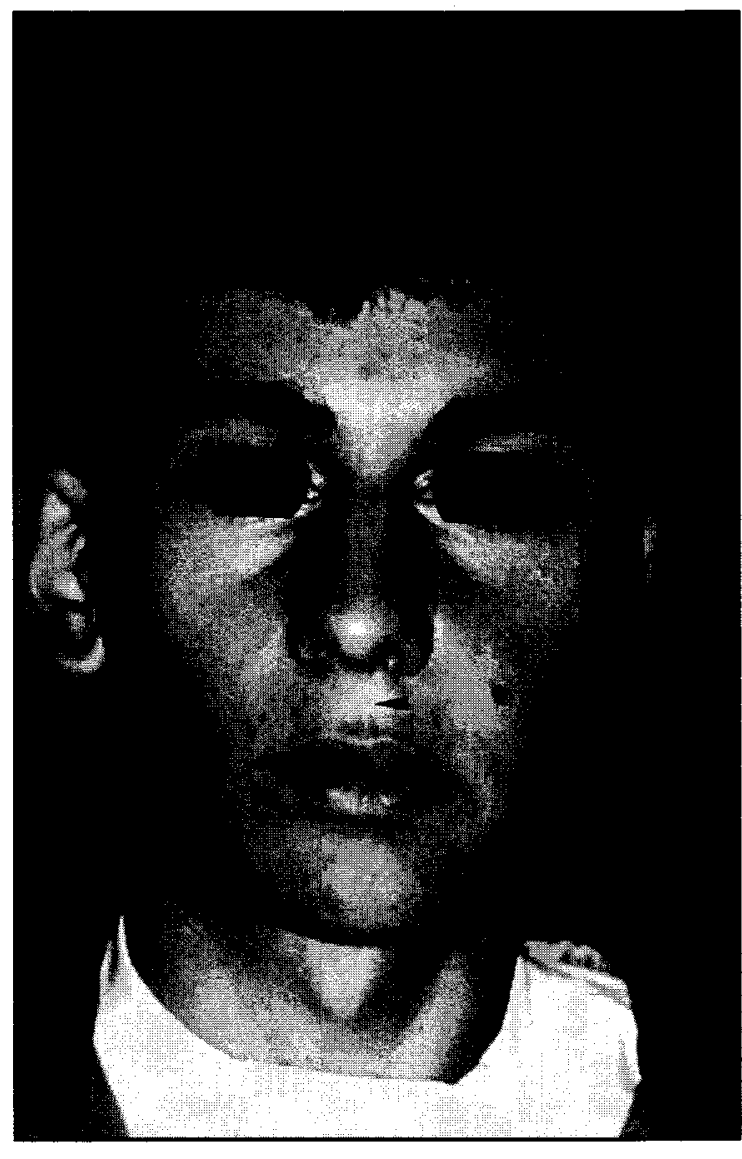

Figura 1 - Aspecto clínico do paciente apresentando aumento de volume abrangendo a hemiface esquerda. Pode-se ainda observar (setas) uma cicatriz de incisão tipo Weber-Fergunson. 


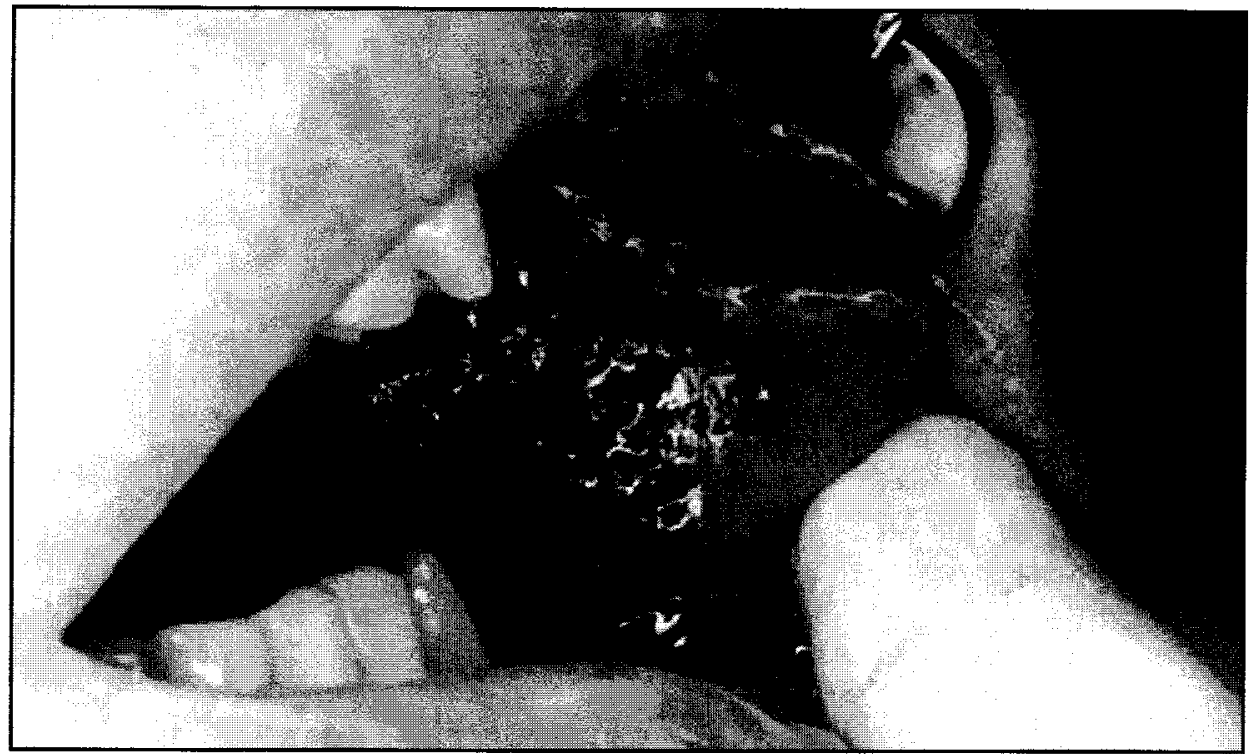

Figura 2 - Aspecto clínico intra-bucal. A mucosa jugal esquerda apresenta edema e superfície com múltiplas vesículas.
Figura 3 - A - Ressonância Magnética (RM), corte coronal. A lesão (setas) aparece expansiva e heterogênea à injeção do contraste, envolvendo a porçăo superior do ramo e côndilo mandibular esquerdos. B - RM, corte axial. A lesão aparece expandindo-se de forma irregular, atingindo o espaço retro-maxilar (a), estendendo-se pela fossa temporal (b) e deformando a parede do seio maxilar (c) esquerdos.

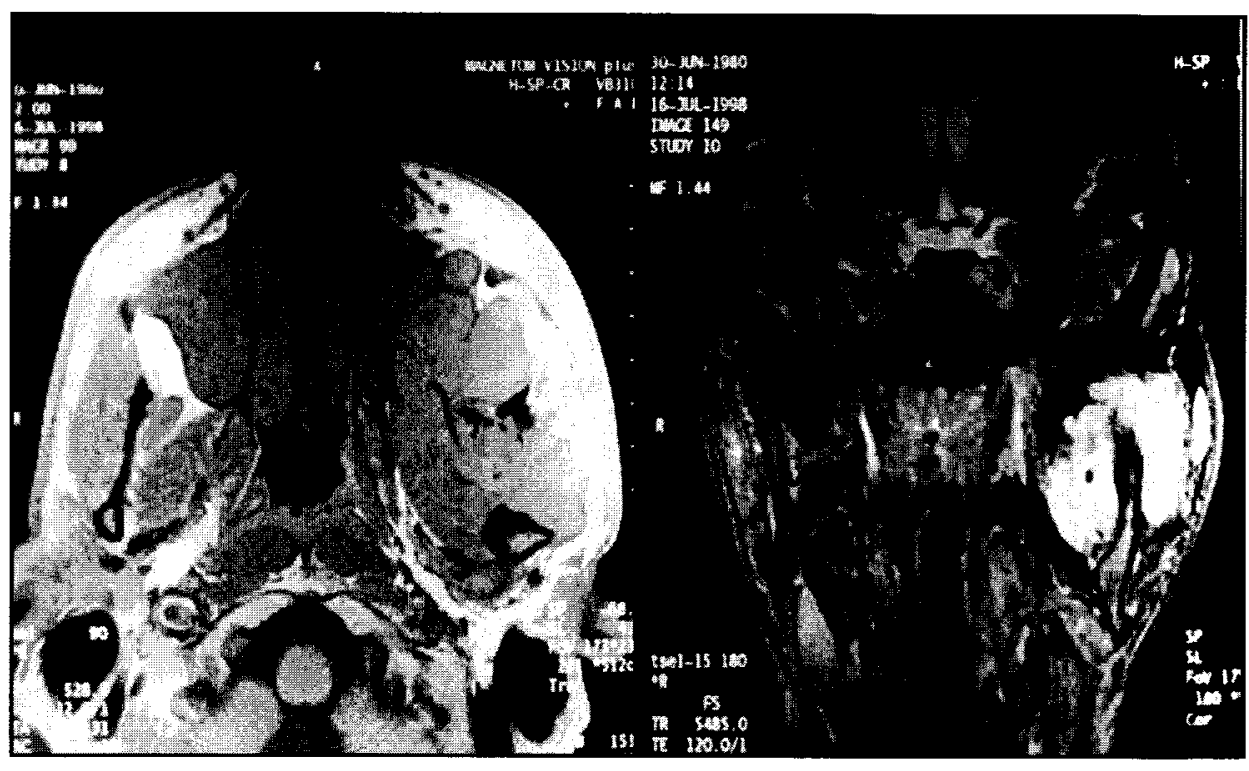

Figura 4 - Fotomicrografia do linfangioma cavernoso, onde visualizam-se vasos linfáticos dilatados, com paredes finas, preenchidos por linfa e algumas células sangüíneas. Aumento de $400 \mathrm{X}$.

\section{ABSTRACT}

The authors present a case of a 18 (eighteen) years old male patient, presenting a recurrent painless tumour on the left side of his face, growing both intra and extraorally. It caused progressive limitation of mandibular mobility. Histopathological result of cavernous lymphangioma differed from the previous findings seen on magnetic resonance imaging.

\section{KEYWORDS}

Cavernous lymphangioma, adult recurrence, magnetic resonance imaging, biopsy

R. Fac. Odontol., Porto Alegre, v. 40, n. I, p. 11-15, set. 1999 


\section{REFERÊNCLAS BIBLIOGRÁFICAS}

1. ARAÚJO, N. S. ; ARAÚJO, V. C. Patologia Bucal. Rio de Janeiro: Artes Médicas, 1984.

2. BAUER, R A.; HARDMAN, F G. Intra-oral Surgical Management of Cystic Hygroma. Brit. J. Oral Surg, n 1, v.14, p.36-40, July, 1976.

3. BHASKAR, S. N. Patologia bucal. São Paulo: Artes Médicas, 1989. p.447.

4. BRENNAN, T. D.; MILLER, A. S.; CHEN, S Lymphangiomas of the Oral Cavity: a clinicopathologic, imunohistochemical, and eletron-microscopic study. J. Oral Maxillofac. Surg., v. 55, n 9, p.932-935, sept. 1997.

5. BÓRAKS, S. Diagnóstico bucal. São Paulo: Artes Médicas, 1996. p.132.

6. CASTRO, A. L. Estomatologia. 2.ed. São Paulo: Santos, 1995. p.92

7. CAWSON, A. R.; BINNIE, W. H.; EVESON, J. W Atlas Colorido de Enfermidades da Boca: correlaçōes clínicas e patológicas. 2.ed. São Paulo: Artes Médicas, 1995. p.17-I0.

8. CECCOTI, E. L. Clínica Stomatologica: SIDA, cáncer y otras afecciones. Buenos Aires: Médica Panamericana, 1993. Cap.7. Lesiones pseudotumorales de la mucosa bucal, p.40-41.

9. FRASER, S. E.; CAMPBELL, B.; KAJDACSY BALLA, A. Pathologic Diagnosis: cystic lymphangioma. Arch. Otolaryngol. Head Neck Surg., v. 122, n.8, p.893-894, Aug. 1996.

10. FONKALSRUD, E. Surgical Management of Congenital Malformations of the Lymphatic System. Am J Surg, v. 128, p.152-159, Aug. 1974

11. FUNG, K.; POENARU, D. S.; KAMAL, I. M Impact af Magnetic Resonance Imaging on the Surgical Management of Cystic Hygromas. J. Pediat. Surg, v.33, n.6, p.839-841, June 1998.

12. herRera, A. M.; PLASENCLA, D. P.; BENITO, M. G. et al. Lynphangiomes Cervicaux Chez L'adulte. Description de Deux Cas. Am Otoraryngol Chir Cervicofac., Paris, v.115, p.299-302, 1998.

13. JASPER, R. D.; GOLDBERG, M. H.; ZBOROWSKI, R. G. Lymphangioma and cystic hygroma. Correction of Facial Growth Disharmony and Obstructive Sleep Apnea. J. Oral Maxillofac. Surg., v.18, p.152-154, 1989.

14. JOSEPHSON, P.; VAN WYK, C. Bilateral Symmetrical Lymphangiomas of the Gingiva: a Case Report. J. Periodontol., p. 47-48, Jan. 1984

15. KAZIM, M.; KENNERDELL, J. S.; ROTHFUS, W. et al. Orbital lymphangioma. Correlation Resonance Images and Intraoperative Findings. Ophthalmol., v99, n.10, p.1588-1594, Oct. 1992.

16. MCDANIEL, R. K.; ADCOCK, J. E. Bilateral Simetrical Lymphangioma of the Gingiva. Oral Surg. Oral Med. Oral Patol., v.63, p.224-227, 1987.

17. NEVILIE, B. W.; DAMM, D. D.; ALLEN, C. M. et al., J. E. Oral Maxillofaval Pathology Philadelphia: Saunders Company, 1995. Cap.12, p.395-397.

18. PINDBORG, J. J. Atlas das Doenças da Mucosa Oral São Paulo: Médica Panamericana, 1981 p.114.
19. QIN, Z. P; XIN, Z. F; REN, L. et al. Long-term Results of Intratumorous Bleomycin-A5 Injection for Head and Neck Lymphangioma. Oral Surg Oral Med. Oral Pathol. Oral Radiol. Endodont. v.86, n.2, p. 139-144, Aug. 1998.

20. REGEZI, J. A.; SCIUBBA, J. J. Patologia Bucal correlaçōes clínico-patológicas. Rio de Janeiro: Guanabara Koogan, 1987. p. 144.

21. SAIJO, M.; MUNRO, I. R.; MANCER, K Lymphangioma: a long-term follow-up study. Plastic \& Reconstructive Surgery, v.56, n.6, p.642-65I, Dec. 1975

22. SERRES, L. M.; SIE, K. C.; RICHARDSON, M. A Lymphatic Malformations of the Head and Neck: a proposal for staging. Arch. Otolaryngol Head Neck Surg., v.12, n.5, p.557-582, May 1995.

23. SHAFER, W. G.; HINE, M. K.; LEVY, B. M Tratado de Patologia Bucal. 4.ed. Rio de Janeiro: Guanabara Koogan, 1987. p.147-149.

24. SMITH, R. J. H.; BURKE, D. K.; YUTAKA, S. et al. OK-432 Therapy for Lymphangiomas. Arch Otolaryngol. Head Neck Surg., v. 122, n. I 1, p.1195-1199, Nov. 1996

25. SOM, P. M.; BRANDNEIN, M. Salivari Galnds tumors and tumorlike conditions. In: SOM, P. M.; CURTIN, H. D. Head and neck imaging. 3.ed. Mosby, 1996. v.3. Cap.17, p.823-914.

26. SONIS, S. T.; FAZIO, R. C.; FANG, L. Princípios e Práticas em Medicina Oral. 2.ed. Rio de Janeiro: Guanabara Koogan, 1996. p.371.

27. TOMMAS1, A. F. Diagnóstico em Patologia Bucal. Rio de Janeiro: Artes Médicas, 1995. p.247-248.

28. YONETSU, K; NAKAYAMA, E.; MIWA, $K$, et al Magnetic Resonance Imaging of Oral and Maxillofacial Angiomas. Oral Surg. Oral Med. Oral Patol, v.76, n.6, p.783-789, Dec. 1993.

29. YUH, W. T; BUEHNER, L. S.; KAO, S. C. et al. Magnetic Resonance Imaging of Pediatric head and Neck Cystic Hygromas. Acta. Otol Rhinol Laryngol, v.100, p.737-742, 1990.

30. ZACHARÍADES, N.; KONDOURIS, I lymphangioma of the oral cavity: report of a case. J. Oral Med., v.39, n.1, p.33-34, Jan-Mar. 1984.

31. ZHAO-JU, Z.; YUN-TANG, W.; XUAN-PENG, Z et al. Clinical Application of Angiography of Oral and Maxillofacial Hemangiomas - Clinical Analysis of Seventy Cases. Oral Surg. Oral Med. Oral Patol, v.55, n.5, p. 437-447, 1983. 